UDC 517.548

E. L. SHISHKINA

\title{
ON WEIGHTED GENERALIZED FUNCTIONS ASSOCIATED WITH QUADRATIC FORMS
}

\begin{abstract}
In this article we consider certain types of weighted generalized functions associated with nondegenerate quadratic forms. Such functions and their derivatives are used for constructing fundamental solutions of iterated ultra-hyperbolic equations with the Bessel operator and for constructing negative real powers of ultra-hyperbolic operators with the Bessel operator.
\end{abstract}

Key words: weighted generalized function, quadratic form, ultrahyperbolic operator, Bessel operator

2010 Mathematical Subject Classification: 46T12, 46F05

1. Introduction and main definitions. The weighted generalized functions associated with nondegenerate indefinite quadratic forms considered in this article are necessary for construction of the ultra-hyperbolic Riezs potential with the Bessel operator. Riezs potential with the Bessel operator and other operators with the Bessel differential operator are very interesting subjects with many applications (see, for example, [1]-9]).

We deal with the part of the Euclidean space

$$
\mathbb{R}_{n}^{+}=\left\{x=\left(x_{1}, \ldots, x_{n}\right) \in \mathbb{R}_{n}, x_{1}>0, \ldots, x_{n}>0\right\} .
$$

Let $\Omega$ be finite or infinite open set in $\mathbb{R}_{n}$, symmetric with respect to each hyperplane $x_{i}=0, i=1, \ldots, n, \Omega_{+}=\Omega \cap \mathbb{R}_{n}^{+}$and $\bar{\Omega}_{+}=\Omega \cap \overline{\mathbb{R}}_{n}^{+}$where

$$
\overline{\mathbb{R}}_{n}^{+}=\left\{x=\left(x_{1}, \ldots, x_{n}\right) \in \mathbb{R}_{n}, \quad x_{1} \geq 0, \ldots, x_{n} \geq 0\right\} .
$$

We have $\Omega_{+} \subseteq \mathbb{R}_{n}^{+}$and $\bar{\Omega}_{+} \subseteq \overline{\mathbb{R}}_{n}^{+}$.

We consider the class $C^{\infty}\left(\Omega_{+}\right)$consisting of infinitely differentiable on $\Omega_{+}$functions. We denote the subset of functions from $C^{\infty}\left(\Omega_{+}\right)$such that all derivatives of these functions with respect to $x_{i}$ for any $i=1, \ldots, n$ are 
continuous up to $x_{i}=0$ by $C^{\infty}\left(\bar{\Omega}_{+}\right)$. A function $f \in C^{\infty}\left(\bar{\Omega}_{+}\right)$will be called even with respect to $x_{i}, i=1, \ldots, n$ if $\left.\frac{\partial^{2 k+1} f}{\partial x_{i}^{2 k+1}}\right|_{x=0}=0$ for all nonnegative integer $k$ (see [10, p. 21). Class $C_{e v}^{\infty}\left(\bar{\Omega}_{+}\right)$consists of functions from $C^{\infty}\left(\bar{\Omega}_{+}\right)$, even with respect to each variable $x_{i}, i=1, \ldots, n$. Let $\stackrel{\circ}{C}_{e v}^{\infty}\left(\bar{\Omega}_{+}\right)$ be the space of all functions $f \in C^{\infty}\left(\bar{\Omega}_{+}\right)$with a compact support. We will call elements of $\stackrel{\circ}{C}_{e v}^{\infty}\left(\bar{\Omega}_{+}\right)$test functions and use the notation $\stackrel{\circ}{C}_{e v}^{\infty}\left(\bar{\Omega}_{+}\right)=$ $=\mathcal{D}_{+}\left(\bar{\Omega}_{+}\right)$.

We define $K$ as an arbitrary compact in $\mathbb{R}_{n}$ symmetric with respect to each hyperplane $x_{i}=0, i=1, \ldots, n, K_{+}=K \cap \overline{\mathbb{R}}_{n}^{+}$. A distribution u on $\bar{\Omega}_{+}$is a linear form on $\mathcal{D}_{+}\left(\bar{\Omega}_{+}\right)$such that for all compacts $K_{+} \subset \bar{\Omega}_{+}$, constants $C$ and $k$ exist and

$$
|u(f)| \leq C \sum_{|\alpha| \leq k} \sup \left|D^{\alpha} f\right|, \quad f \in \stackrel{\circ}{C}_{e v}^{\infty}\left(K_{+}\right),
$$

where $D^{\alpha}=D_{x_{1}}^{\alpha_{1}} \ldots D_{x_{n}}^{\alpha_{n}}, \alpha=\left(\alpha_{1}, \ldots, \alpha_{n}\right), \alpha_{1}, \ldots, \alpha_{n}$ are integer nonnegative numbers, $D_{x_{j}}=i \frac{\partial}{\partial x_{j}}, i$ is imaginary unit, $j=1, \ldots, n$. The set of all distributions on the set $\bar{\Omega}_{+}$is denoted by $\mathcal{D}_{+}^{\prime}\left(\bar{\Omega}_{+}\right)$(see [10], p. 11 and [11, p. 34).

Multiindex $\gamma=\left(\gamma_{1}, \ldots, \gamma_{n}\right)$ consists of positive fixed reals $\gamma_{i}>0$, $i=1, \ldots, n$ and $|\gamma|=\gamma_{1}+\ldots+\gamma_{n}$. Let $L_{p}^{\gamma}\left(\Omega_{+}\right), 1 \leq p<\infty$ be the space of all measurable in $\Omega_{+}$functions even with respect to each variable $x_{i}$, $i=1, \ldots, n$ such that

$$
\int_{\Omega_{+}}|f(x)|^{p} x^{\gamma} d x<\infty, \quad x^{\gamma}=\prod_{i=1}^{n} x_{i}^{\gamma_{i}} .
$$

For a real number $p \geq 1$, the $L_{p}^{\gamma}\left(\Omega_{+}\right)$-norm of $f$ is defined by

$$
\|f\|_{L_{p}^{\gamma}\left(\Omega_{+}\right)}=\left(\int_{\Omega_{+}}|f(x)|^{p} x^{\gamma} d x\right)^{1 / p} .
$$

Weighted measure of $\Omega_{+}$is denoted by $\operatorname{mes}_{\gamma}(\Omega)$ and is defined by formula

$$
\operatorname{mes}_{\gamma}\left(\Omega_{+}\right)=\int_{\Omega_{+}} x^{\gamma} d x
$$


For every measurable function $f(x)$ defined on $\mathbb{R}_{n}^{+}$we consider

$$
\mu_{\gamma}(f, t)=\operatorname{mes}_{\gamma}\left\{x \in \mathbb{R}_{n}^{+}:|f(x)|>t\right\}=\int_{\{x:|f(x)|>t\}^{+}} x^{\gamma} d x
$$

where $\{x:|f(x)|>t\}^{+}=\left\{x \in \mathbb{R}_{n}^{+}:|f(x)|>t\right\}$. We will call the function $\mu_{\gamma}=\mu_{\gamma}(f, t)$ a weighted distribution function $|f(x)|$.

A space $L_{\infty}^{\gamma}\left(\Omega_{+}\right)$is defined as a set of measurable on $\Omega_{+}$and even with respect to each variable functions $f(x)$ such as

$$
\|f\|_{L_{\infty}^{\gamma}\left(\Omega_{+}\right)}=\operatorname{esssup}_{x \in \Omega_{+}}|f(x)|=\inf _{a \in \Omega_{+}}\left\{\mu_{\gamma}(f, a)=0\right\}<\infty .
$$

For $1 \leq p \leq \infty$ the $L_{p, l o c}^{\gamma}\left(\Omega_{+}\right)$is the set of functions $u(x)$ defined almost everywhere in $\Omega_{+}$such that $u f \in L_{p}^{\gamma}\left(\Omega_{+}\right)$for any $f \in \stackrel{\circ}{C}_{e v}^{\infty}\left(\bar{\Omega}_{+}\right)$. Each function $u(x) \in L_{1, l o c}^{\gamma}\left(\Omega_{+}\right)$will be identified with the functional $u \in$ $\in \mathcal{D}_{+}^{\prime}\left(\bar{\Omega}_{+}\right)$acting according to the formula

$$
(u, f)_{\gamma}=\int_{\mathbb{R}_{n}^{+}} u(x) f(x) x^{\gamma} d x, \quad x^{\gamma}=\prod_{i=1}^{n} x_{i}^{\gamma_{i}}, \quad f \in \stackrel{\circ}{C}_{e v}^{\infty}\left(\overline{\mathbb{R}}_{n}^{+}\right) .
$$

Functionals $u \in \mathcal{D}_{+}^{\prime}\left(\bar{\Omega}_{+}\right)$acting by the formula $(1)$ will be called regular weighted functionals. All other functionals $u \in \mathcal{D}_{+}^{\prime}\left(\bar{\Omega}_{+}\right)$will be called singular weighted functionals.

\section{Weighted generalized functions concentrated on the part} of the cone. In this section we consider weighted generalized functions $\delta_{\gamma}(P)$ concentrated on the part of the cone and give formulas for their derivatives.

Generalized function $\delta_{\gamma}$ is defined by equality (by analogy with [12] p. 247)

$$
\left(\delta_{\gamma}, \varphi\right)_{\gamma}=\varphi(0), \quad \varphi(x) \in K^{+} .
$$

For convenience we will write

$$
\left(\delta_{\gamma}, \varphi\right)_{\gamma}=\int_{\mathbb{R}_{n}^{+}} \delta_{\gamma}(x) \varphi(x) x^{\gamma} d x=\varphi(0) .
$$

Let $p, q \in \mathbb{N}, n=p+q$ and

$$
P=\left|x^{\prime}\right|^{2}-\left|x^{\prime \prime}\right|^{2}=x_{1}^{2}+\ldots+x_{p}^{2}-x_{p+1}^{2}-\ldots-x_{p+q}^{2},
$$


where $x=\left(x_{1}, \ldots, x_{n}\right)=\left(x^{\prime}, x^{\prime \prime}\right) \in \mathbb{R}_{n}^{+}, x^{\prime}=\left(x_{1}, \ldots, x_{p}\right), x^{\prime \prime}=\left(x_{p+1}, \ldots, x_{p+q}\right)$.

Definition 1. Let $\varphi \in \mathcal{D}_{+}\left(\overline{\mathbb{R}}_{n}^{+}\right)$vanishes at the origin. For such $\varphi$ we define generalized function $\delta_{\gamma}(P)$ concentrated on the part of the cone $P=0$ belonging to $\mathbb{R}_{n}^{+}$by the formula

$$
\left(\delta_{\gamma}(P), \varphi\right)_{\gamma}=\int_{\mathbb{R}_{n}^{+}} \delta_{\gamma}\left(\left|x^{\prime}\right|^{2}-\left|x^{\prime \prime}\right|^{2}\right) \varphi(x) x^{\gamma} d x .
$$

If the function $\varphi \in \mathcal{D}_{+}\left(\overline{\mathbb{R}}_{n}^{+}\right)$does not vanish at the origin then $\left(\delta_{\gamma}(P), \varphi\right)_{\gamma}$ is defined by regularizing the integral.

Lemma 1. Let $\varphi \in \mathcal{D}_{+}\left(\overline{\mathbb{R}}_{n}^{+}\right)$vanishes at the origin, $p>1$ and $q>1$. For $\delta_{\gamma}(P)$ the representation

$$
\left(\delta_{\gamma}(P), \varphi\right)_{\gamma}=\frac{1}{2} \int_{0}^{\infty} \int_{S_{p}^{+}} \int_{S_{q}^{+}} \varphi(s \omega) s^{n+|\gamma|-3} \omega^{\gamma} d S_{p} d S_{q} d s
$$

holds true. In (3) $\omega=\left(\omega^{\prime}, \omega^{\prime \prime}\right), \omega^{\prime}=\left(\omega_{1}, \ldots, \omega_{p}\right) \in \mathbb{R}_{p}^{+}, \omega^{\prime \prime}=\left(\omega_{p+1}, \ldots, \omega_{p+q}\right) \in$ $\in \mathbb{R}_{q}^{+}, n=p+q,\left|\omega^{\prime}\right|=\left|\omega^{\prime \prime}\right|=1, \omega^{\gamma}=\prod_{i=1}^{n} \omega_{i}^{\gamma_{i}}, d S_{p}$ and $d S_{q}$ are elements of surface area on the part of the unit sphere

$$
S_{p}^{+}=\left\{\omega^{\prime} \in \mathbb{R}_{p}^{+}:\left|\omega^{\prime}\right|=1\right\} \quad S_{q}^{+}=\left\{\omega^{\prime \prime} \in \mathbb{R}_{q}^{+}:\left|\omega^{\prime \prime}\right|=1\right\},
$$

respectively. For the $k$-th derivative $(k \in \mathbb{N})$ of $\delta_{\gamma}(P)$ we have

$$
\left(\delta_{\gamma}^{(k)}(P), \varphi\right)_{\gamma}=\int_{0}^{\infty}\left[\left(\frac{1}{2 s} \frac{\partial}{\partial s}\right)^{k} \psi(r, s) s^{q+\left|\gamma^{\prime \prime}\right|-2}\right]_{s=r} r^{p+\left|\gamma^{\prime}\right|-1} d r,
$$

where

$$
\psi(r, s)=\frac{1}{2} \int_{S_{p}^{+}} \int_{S_{q}^{+}} \varphi\left(r \omega^{\prime}, s \omega^{\prime \prime}\right) \omega^{\gamma} d S_{p} d S_{q} .
$$

Proof. Let us transform (2) to bipolar coordinates defined by

$$
x_{1}=r \omega_{1}, \ldots, x_{p}=r \omega_{p}, x_{p+1}=s \omega_{p+1}, \ldots, x_{p+q}=s \omega_{p+q},
$$

where

$$
r=\sqrt{x_{1}^{2}+\ldots+x_{p}^{2}}, \quad s=\sqrt{x_{p+1}^{2}+\ldots+x_{p+q}^{2}},
$$




$$
\left|\omega^{\prime}\right|=\sqrt{\omega_{1}^{2}+\ldots+\omega_{p}^{2}}=1, \quad\left|\omega^{\prime \prime}\right|=\sqrt{\omega_{p+1}^{2}+\ldots+\omega_{p+q}^{2}}=1 .
$$

We obtain

$$
\left(\delta_{\gamma}(P), \varphi(x)\right)_{\gamma}=
$$

$$
=\int_{0}^{\infty} \int_{0}^{\infty} \int_{S_{p}^{+}} \int_{S_{q}^{+}} \delta_{\gamma}\left(r^{2}-s^{2}\right) \varphi\left(r \omega^{\prime}, s \omega^{\prime \prime}\right) r^{p+\left|\gamma^{\prime}\right|-1} s^{q+\left|\gamma^{\prime \prime}\right|-1} \omega^{\gamma} d S_{1}^{p} d S_{1}^{q} d r d s .
$$

Now let us choose the coordinates to be $r^{2}=u, s^{2}=v$. In these coordinates we have

$$
\begin{gathered}
\left(\delta_{\gamma}(P), \varphi\right)_{\gamma}=\frac{1}{4} \int_{0}^{\infty} \int_{0}^{\infty} \int_{S_{p}^{+}} \int_{S_{q}^{+}} \delta_{\gamma}(u-v) \varphi\left(\sqrt{u} \omega^{\prime}, \sqrt{v} \omega^{\prime \prime}\right) u^{\frac{p+\left|\gamma^{\prime}\right|}{2}-1} \times \\
\times v^{\frac{q+\left|\gamma^{\prime \prime}\right|}{2}-1} \omega^{\gamma} d S_{p} d S_{q} d u d v=\frac{1}{4} \int_{0}^{\infty} \int_{S_{p}^{+}} \int_{S_{q}^{+}} \varphi(\sqrt{v} \omega) v^{\frac{n+|\gamma|}{2}-2} \omega^{\gamma} d S_{p} d S_{q} d v .
\end{gathered}
$$

Returning to variable $s$ by the formula $v=s^{2}$, we obtain (3).

Now we prove the formula (4). After the change of variables by (6) and $r^{2}=u, s^{2}=v$ in $\left(\delta_{\gamma}^{(k)}(P), \varphi\right)_{\gamma}$ we get

$$
\begin{gathered}
\left(\delta_{\gamma}^{(k)}(P), \varphi\right)_{\gamma}=\frac{1}{4} \int_{0}^{\infty} \int_{0}^{\infty} \int_{S_{p}^{+}} \int_{S_{q}^{+}} \frac{\partial^{k}}{\partial v^{k}}\left[\delta_{\gamma}(v-u)\right] \varphi\left(\sqrt{u} \omega^{\prime}, \sqrt{v} \omega^{\prime \prime}\right) \times \\
\times u^{\frac{p+\left|\gamma^{\prime}\right|}{2}-1} v^{\frac{q+\left|\gamma^{\prime \prime}\right|}{2}-1} \omega^{\gamma} d S_{p} d S_{q} d u d v=\int_{0}^{\infty} \int_{0}^{\infty} \int_{S_{p}^{+}} \int_{S_{q}^{+}} \delta_{\gamma}(v-u) \times \\
\times \frac{(-1)^{k}}{4} \frac{\partial^{k}}{\partial v^{k}}\left[\varphi\left(\sqrt{u} \omega^{\prime}, \sqrt{v} \omega^{\prime \prime}\right) v^{\frac{q+\left|\gamma^{\prime \prime}\right|}{2}}-1\right] u^{\frac{p+\left|\gamma^{\prime}\right|}{2}-1} \omega^{\gamma} d S_{p} d S_{q} d u d v= \\
=\frac{(-1)^{k}}{4} \int_{0}^{\infty} \int_{S_{p}^{+}}^{\infty} \int_{S_{q}^{+}} u^{\frac{p+\left|\gamma^{\prime}\right|}{2}-1} \omega^{\gamma}\left[\frac{\partial^{k}}{\partial v^{k}} \varphi\left(\sqrt{u} \omega^{\prime}, \sqrt{v} \omega^{\prime \prime}\right) v^{\frac{q+\left|\gamma^{\prime \prime}\right|}{2}}-1\right]_{v=u} d S_{p} d S_{q} d u .
\end{gathered}
$$

Returning to variables $r, s$ and using notation (5) we obtain (4). This completes the proof of Lemma 1. 
Remark 1. Similarly, we can get the formula

$$
\left(\delta_{\gamma}^{(k)}(P), \varphi\right)_{\gamma}=(-1)^{k} \int_{0}^{\infty}\left[\left(\frac{1}{2 r} \frac{\partial}{\partial r}\right)^{k} \psi(r, s) r^{p+\left|\gamma^{\prime}\right|-2}\right]_{r=s} s^{q+\left|\gamma^{\prime \prime}\right|-1} d s .
$$

Remark 2. Noticing that when $k=0$ formulas (4) and (7) are equivalent to the formula (3) we will examine intergals (4) and (7) at $k \in \mathbb{N} \cup\{0\}$.

Let $\varphi \in \mathcal{D}_{+}\left(\overline{\mathbb{R}}_{n}^{+}\right)$. Assuming that the function $\varphi$ vanishes at the origin we have that integrals (4) and (7) converge for all $k \in \mathbb{N} \cup\{0\}$. If the function $\varphi$ does not vanish at the origin then integrals (4) and (7) converge only for $k<\frac{p+q+|\gamma|-2}{2}$. In this case for $k \geq \frac{p+q+|\gamma|-2}{2}$ we will consider the regularization of (4) and (7) denoting them $\delta_{\gamma, 1}^{(k)}(P)$ and $\delta_{\gamma, 2}^{(k)}(P)$, respectively. So using the expression (5) for $p>1, q>1$ and $k \in \mathbb{N} \cup\{0\}$ we have

$$
\begin{gathered}
\left(\delta_{\gamma, 1}^{(k)}(P), \varphi\right)_{\gamma}=\left.\int_{0}^{+\infty}\left[\left(\frac{1}{2 s} \frac{\partial}{\partial s}\right)^{k} \psi(r, s) s^{q+\left|\gamma^{\prime \prime}\right|-2}\right]\right|_{s=r} r^{p+\left|\gamma^{\prime}\right|-1} d r \\
\left(\delta_{\gamma, 2}^{(k)}(P), \varphi\right)_{\gamma}=\left.(-1)^{k} \int_{0}^{+\infty}\left[\left(\frac{1}{2 r} \frac{\partial}{\partial r}\right)^{k} \psi(r, s) r^{p+\left|\gamma^{\prime}\right|-2}\right]\right|_{r=s} s^{q+\left|\gamma^{\prime \prime}\right|-1} d s .
\end{gathered}
$$

The integrals (8) and (9) converge and coincide for $k<\frac{p+q+|\gamma|-2}{2}$ and for $k \geq \frac{p+q+|\gamma|-2}{2}$ these integrals must be understood in the sense of their regularizations.

2.Weighted generalized function $P_{\gamma,+}^{\lambda}$. Let $n=p+q, p>1, q>1$ and $P(x)=x_{1}^{2}+\ldots+x_{p}^{2}-x_{p+1}^{2}-\ldots-x_{p+q}^{2}$. Here and further let $\varphi \in \mathcal{D}_{+}\left(\overline{\mathbb{R}}_{n}^{+}\right)$. We define the weighted generalized function $P_{\gamma,+}^{\lambda}$ by

$$
\left(P_{\gamma,+}^{\lambda}, \varphi\right)_{\gamma}=\int_{\{P(x)>0\}^{+}} P^{\lambda}(x) \varphi(x) x^{\gamma} d x
$$

where $\{P(x)>0\}^{+}=\left\{x \in \mathbb{R}_{n}^{+}: P(x)>0\right\}, \lambda \in \mathbb{C}$.

Weighted generalized function $P_{\gamma,+}^{\lambda}$ and its derivatives are used for constructing fundamental solutions of iterated B-ultra-hyperbolic equations of the form $L_{B}^{k} u=f(x), \quad k \in \mathbb{N}, \quad x \in \mathbb{R}_{n}, \quad x_{i}>0, \quad i=1, \ldots, n$, 
where $L_{B}$ is B-ultra-hyperbolic operator (see [9] and [13]-[15])

$$
L_{B}=B_{x_{1}}+\ldots+B_{x_{p}}-B_{x_{p+1}}-\ldots-B_{x_{n}}
$$

$B_{x_{i}}=\frac{\partial^{2}}{\partial x_{i}^{2}}+\frac{\gamma_{i}}{x_{i}} \frac{\partial}{\partial x_{i}}$ is the Bessel operator, $\gamma_{i}>0, i=1, \ldots, n$.

It should also be noted that negative real powers of an operator $L_{B}$ called generalized B-hyperbolic potentials (see [16]) are constructed using function $P_{\gamma,+}^{\lambda}$. Let us find singularities of $\left(P_{\gamma,+}^{\lambda}, \varphi\right)_{\gamma}$. For this purpose we transform (10) to bipolar coordinates (6) and using notation (5) for integral 10 we obtain

$$
\left(P_{\gamma,+}^{\lambda}, \varphi\right)_{\gamma}=\int_{0}^{\infty} \int_{0}^{r}\left(r^{2}-s^{2}\right)^{\lambda} \psi(r, s) r^{p+\left|\gamma^{\prime}\right|-1} s^{q+\left|\gamma^{\prime \prime}\right|-1} d r d s .
$$

We now make change of variables $u=r^{2}, v=s^{2}$ in 11 :

$$
\left(P_{\gamma,+}^{\lambda}, \varphi\right)_{\gamma}=\frac{1}{4} \int_{0}^{\infty} \int_{0}^{u}(u-v)^{\lambda} \psi_{1}(u, v) u^{\frac{p+\left|\gamma^{\prime}\right|}{2}-1} s^{\frac{q+\left|\gamma^{\prime \prime}\right|}{2}-1} d u d v
$$

where $\psi_{1}(u, v)=\psi(r, s)$ when $u=r^{2}, v=s^{2}$.

If we write $v=u t$ then we obtain

$$
\left(P_{\gamma,+}^{\lambda}, \varphi\right)_{\gamma}=\int_{0}^{\infty} u^{\lambda+\frac{p+q+|\gamma|}{2}-1} \Phi(\lambda, u) d u
$$

where

$$
\Phi(\lambda, u)=\frac{1}{4} \int_{0}^{1}(1-t)^{\lambda} t^{\frac{q+\left|\gamma^{\prime \prime}\right|}{2}}-1 \psi_{1}(u, t u) d t .
$$

The formula 12 shows that $P_{\gamma,+}^{\lambda}$ has two sets of poles. The first consists of poles of $\Phi(\lambda, u)$. Namely for $t=1$ function $\Phi(\lambda, u)$ has singularity when

$$
\lambda=-1,-2, \ldots,-k, \ldots
$$

in which $\Phi(\lambda, u)$ has simple poles with residues

$$
\underset{\lambda=-k}{\operatorname{res}} \Phi(\lambda, u)=\frac{1}{4} \frac{(-1)^{k-1}}{(k-1) !} \frac{\partial^{k-1}}{\partial t^{k-1}}\left[t^{\frac{q+\left|\gamma^{\prime \prime}\right|-2}{2}} \psi_{1}(u, t u)\right]_{t=1} .
$$


Moreover integral 12$)$ has poles at the points

$$
\lambda=-\frac{n+|\gamma|}{2},-\frac{n+|\gamma|}{2}-1, \ldots,-\frac{n+|\gamma|}{2}-k, \ldots
$$

where $n=p+q, \gamma=\left(\gamma^{\prime}, \gamma^{\prime \prime}\right)$. Wherein

$$
\underset{\lambda=-\frac{n+|\gamma|}{2}-k}{\operatorname{res}}\left(P_{\gamma,+}^{\lambda}, \varphi\right)_{\gamma}=\left.\frac{1}{k !} \frac{\partial^{k}}{\partial u^{k}} \Phi\left(-\frac{n+|\gamma|}{2}-k, u\right)\right|_{u=0} .
$$

We have three cases. The first case is when a singular point $\lambda$ belongs to the first set (14), but not to the second (16). The second case is when singular point $\lambda$ belongs to the second $(16)$, but $\lambda \neq-k, k \in \mathbb{N}$. And the third case is when $\lambda$ belongs both to the first set (14) and the second set (16). Let us now study each case separately in the following three theorems.

Theorem 1. If $\lambda=-k, k \in \mathbb{N}$ and $n+|\gamma| \in \mathbb{R} \backslash \mathbb{N}$ or $n+|\gamma| \in \mathbb{N}$ and $n+|\gamma|=2 k-1, k \in \mathbb{N}$ and also if $n+|\gamma|$ is even and $k<\frac{n+|\gamma|}{2}$ the weighted generalized function $P_{\gamma,+}^{\lambda}$ has simple pole with residue

$$
\underset{\lambda=-k}{\operatorname{res}} P_{\gamma,+}^{\lambda}=\frac{(-1)^{k-1}}{(k-1) !} \delta_{\gamma, 1}^{(k-1)}(P) .
$$

Proof. Let us write $\Phi(\lambda, u)$ in the neighborhood of $\lambda=-k$ in the form

$$
\Phi(\lambda, u)=\frac{\Phi_{0}(u)}{\lambda+k}+\Phi_{1}(\lambda, u), \quad \Phi_{0}(u)=\underset{\lambda=-k}{\operatorname{res}} \Phi(\lambda, u),
$$

where function $\Phi_{1}(\lambda, u)$ is regular at $\lambda=-k$. We obtain

$$
\left(P_{\gamma,+}^{\lambda}, \varphi\right)_{\gamma}=\frac{1}{\lambda+k} \int_{0}^{\infty} u^{\lambda+\frac{n+|\gamma|}{2}-1} \Phi_{0}(u) d u+\int_{0}^{\infty} u^{\lambda+\frac{n+|\gamma|}{2}-1} \Phi_{1}(\lambda, u) d u .
$$

The integrals in (19) are regular functions of $\lambda$ at $\lambda=-k$. Therefore $\left(P_{\gamma,+}^{\lambda}, \varphi\right)_{\gamma}$ has a simple pole at such a point and using 15 we have

$$
\underset{\lambda=-k}{\operatorname{res}}\left(P_{\gamma,+}^{\lambda}, \varphi\right)=\frac{(-1)^{k-1}}{4(k-1) !} \int_{0}^{\infty} u^{\frac{n+|\gamma|}{2}-k-1} \frac{\partial^{k-1}}{\partial t^{k-1}}\left[t^{\frac{q+\left|\gamma^{\prime \prime}\right|}{2}-1} \psi_{1}(u, t u)\right]_{t=1} d u .
$$


If in 20 we get $t u=v$ then we may write

$$
\operatorname{res}_{\lambda=-k}\left(P_{\gamma,+}^{\lambda}, \varphi\right)=\frac{(-1)^{k-1}}{4(k-1) !} \int_{0}^{\infty} \frac{\partial^{k-1}}{\partial v^{k-1}}\left[v^{\frac{q+\left|\gamma^{\prime \prime}\right|}{2}-1} \psi_{1}(u, v)\right]_{v=u} u^{\frac{p+\left|\gamma^{\prime}\right|}{2}-1} d u
$$

where the integral is to be understood in the sense of its regularization for $k \geq \frac{n}{2}$. We now make the change of variables $u=r^{2}$ and $v=s^{2}$ in (8) and have

$$
\left(\delta_{\gamma, 1}^{(k-1)}(P), \varphi\right)_{\gamma}=\frac{1}{2} \int_{0}^{\infty}\left[\frac{\partial^{k-1}}{\partial v^{k-1}} v^{\frac{q+\left|\gamma^{\prime \prime}\right|}{2}-1} \psi_{1}(u, v)\right]_{v=u} u^{\frac{p+\left|\gamma^{\prime}\right|}{2}-1} d u,
$$

where

$$
\psi_{1}(u, v)=\frac{1}{2} \int_{S_{p}^{+}} \int_{S_{q}^{+}} \varphi\left(\sqrt{u} \omega^{\prime}, \sqrt{v} \omega^{\prime \prime}\right) \omega^{\gamma} d S_{p} d S_{q} .
$$

Formulas (21) and 22 imply (17). For $k \geq \frac{n}{2}$ integral in 22 is to be understood in the sense of its regularization. In the case when $n+|\gamma| \in \mathbb{R} \backslash \mathbb{N}$ or $n+|\gamma| \in \mathbb{N}$ and $n+|\gamma|=2 k-1, k \in \mathbb{N}$ regularization of the integral in $(22)$ is defined by analytic continuation. This proves the desired result.

Now we study the case when the singular point $\lambda$ is in the second set (16), but not in the first (14). If $\lambda=-\frac{n+|\gamma|}{2}-k, k=0,1,2, \ldots$, and $n+|\gamma| \in \mathbb{R} \backslash \mathbb{N}$ or $n+|\gamma| \in \mathbb{N}$ and $n+|\gamma|=2 k-1, k \in \mathbb{N}$, then function $\Phi(\lambda, u)$ is regular in the neighborhood of $\lambda=-\frac{n+|\gamma|}{2}-k$. Therefore function $\left(P_{\gamma,+}^{\lambda}, \varphi\right)_{\gamma}$ will have a simple pole with residue given by (17).

Before proceeding to the expression of the residue $\underset{\lambda=-\frac{n+|\gamma|}{2}-k}{\operatorname{res}}\left(P_{\gamma,+}^{\lambda}, \varphi\right)$ through derivatives of function $\varphi(x)$ at the origin we will obtain one useful formula. Consider the B-ultra-hyperbolic differential operator

$$
L_{B}=B_{\gamma_{1}^{\prime}}+\ldots+B_{\gamma_{p}^{\prime}}-B_{\gamma_{p+1}^{\prime \prime}}-B_{\gamma_{p+q}^{\prime \prime}}, \quad B_{\gamma_{i}}=\frac{\partial^{2}}{\partial x_{i}^{2}}+\frac{\gamma_{i}}{x_{i}} \frac{\partial}{\partial x_{i}} .
$$

Applying an operator $L_{B}$ to quadratic form

$$
P(x)=x_{1}^{2}+\ldots+x_{p}^{2}-x_{p+1}^{2}-\ldots-x_{p+q}^{2}, n=p+q, p>1, q>1
$$

we obtain

$$
L_{B} P^{\lambda+1}(x)=4(\lambda+1)\left(\lambda+\frac{n+|\gamma|}{2}\right) P^{\lambda}(x) .
$$


Theorem 2. Let $n+|\gamma|$ be not integer or $n+|\gamma| \in \mathbb{N}$ and $n+|\gamma|=2 k-1$, $k \in \mathbb{N}$. When $p+\left|\gamma^{\prime}\right|$ is not integer or $p+\left|\gamma^{\prime}\right| \in \mathbb{N}, p+\left|\gamma^{\prime}\right|=2 m-1, m \in \mathbb{N}$ and $q+\left|\gamma^{\prime \prime}\right|$ is even weighted functional $P_{\gamma,+}^{\lambda}$ has simple poles at $\lambda=-\frac{n+|\gamma|}{2}-k$, $k \in \mathbb{N} \cup\{0\}$ with residues

$$
\operatorname{res}_{\lambda=-\frac{n+|\gamma|}{2}-k} P_{\gamma,+}^{\lambda}=\frac{(-1)^{\frac{q+\left|\gamma^{\prime \prime}\right|}{2}}}{2^{n+2 k} k !} \frac{\prod_{i=1}^{n} \Gamma\left(\frac{\gamma_{i}+1}{2}\right)}{\Gamma\left(\frac{n+|\gamma|}{2}+k\right)} L_{B}^{k} \delta_{\gamma}(x) .
$$

If $p+\left|\gamma^{\prime}\right|$ is even then weighted functional $P_{\gamma,+}^{\lambda}$ is regular at $\lambda=-\frac{n+|\gamma|}{2}-k$, $k \in \mathbb{N} \cup\{0\}$.

Proof. We first consider $\lambda=-\frac{n+|\gamma|}{2}$. Using formula (17) we can write

$$
\begin{gathered}
\underset{\substack{\lambda=-\frac{n+|\gamma|}{2} \\
\operatorname{res}}}{\lambda}\left(P_{\gamma,+}^{\lambda}, \varphi\right)_{\gamma}=\Phi\left(-\frac{n+|\gamma|}{2}, 0\right)=\frac{\psi_{1}(0,0)}{4} \int_{0}^{1}(1-t)^{-\frac{n+|\gamma|}{2} t^{\frac{q+\left|\gamma^{\prime \prime}\right|}{2}} d t=} \\
=\frac{1}{4} \psi_{1}(0,0) \frac{\Gamma\left(\frac{q+\left|\gamma^{\prime \prime}\right|}{2}\right) \Gamma\left(-\frac{n+|\gamma|}{2}+1\right)}{\Gamma\left(-\frac{p+\left|\gamma^{\prime}\right|}{2}+1\right)} .
\end{gathered}
$$

From the last formula we can see that if $p+\left|\gamma^{\prime}\right|$ is even then

$$
\underset{\lambda=-\frac{n+|\gamma|}{2}}{\operatorname{res}}\left(P_{\gamma,+}^{\lambda}, \varphi\right)=0 .
$$

Now assume that $p+\left|\gamma^{\prime}\right|$ is not integer or $p+\left|\gamma^{\prime}\right| \in \mathbb{N}$ and $p+\left|\gamma^{\prime}\right|=2 k-1$, $k \in \mathbb{N}$ and $q+\left|\gamma^{\prime \prime}\right|$ is even. We have

$$
\psi_{1}(0,0)=\psi(0,0)=\varphi(0) \iint_{S_{p}^{+}} \int_{S_{q}^{+}} \omega^{\gamma} d S_{p} d S_{q}=\varphi(0)\left|S_{1}^{+}(p)\right|_{\gamma^{\prime}}\left|S_{1}^{+}(q)\right|_{\gamma^{\prime \prime}}
$$

where

$$
\left|S_{1}^{+}(p)\right|_{\gamma^{\prime}}=\frac{\prod_{i=1}^{p} \Gamma\left(\frac{\gamma_{i}^{\prime}+1}{2}\right)}{2^{p-1} \Gamma\left(\frac{p+\left|\gamma^{\prime}\right|}{2}\right)}, \quad\left|S_{1}^{+}(q)\right|_{\gamma^{\prime \prime}}=\frac{\prod_{i=1}^{q} \Gamma\left(\frac{\gamma_{i}^{\prime \prime}+1}{2}\right)}{2^{q-1} \Gamma\left(\frac{q+\left|\gamma^{\prime \prime}\right|}{2}\right)}
$$


(see [1], p. 20, formula (1.2.5)). After some simple calculations, we obtain

$$
\operatorname{res}_{\lambda=-\frac{n+|\gamma|}{2}}\left(P_{\gamma,+}^{\lambda}, \varphi\right)_{\gamma}=\frac{(-1)^{\frac{q+\left|\gamma^{\prime \prime}\right|}{2}}}{2^{n}} \frac{\prod_{i=1}^{n} \Gamma\left(\frac{\gamma_{i}+1}{2}\right)}{\Gamma\left(\frac{n+|\gamma|}{2}\right)} \varphi(0) .
$$

Also we have

$$
\operatorname{res}_{\lambda=-\frac{n+|\gamma|}{2}} P_{\gamma,+}^{\lambda}=\frac{(-1)^{\frac{q+\left|\gamma^{\prime \prime}\right|}{2}}}{2^{n}} \frac{\prod_{i=1}^{n} \Gamma\left(\frac{\gamma_{i}+1}{2}\right)}{\Gamma\left(\frac{n+|\gamma|}{2}\right)} \delta_{\gamma}(x) .
$$

Using Green's theorem and formula 23 we derive

$$
\int_{\{P(x)>0\}^{+}}\left(\varphi(x)\left[L_{B} P^{\lambda+1}(x)\right]-P^{\lambda+1}(x)\left[L_{B} \varphi(x)\right]\right) x^{\gamma} d x=0,
$$

therefore

$$
\left(P_{\gamma,+}^{\lambda}, \varphi\right)_{\gamma}=\frac{1}{2(\lambda+1)(2 \lambda+n+|\gamma|)}\left(P_{\gamma,+}^{\lambda+1}, L_{B} \varphi\right)_{\gamma}
$$

Then $k$-fold iteration of 28 leads to

$$
\left(P_{\gamma,+}^{\lambda}, \varphi\right)_{\gamma}=\frac{\left(P_{\gamma,+}^{\lambda+k}, L_{B}^{k} \varphi\right)_{\gamma}}{2^{2 k}(\lambda+1) \ldots(\lambda+k)\left(\lambda+\frac{n+|\gamma|}{2}\right) \ldots\left(\lambda+\frac{n+|\gamma|}{2}+k-1\right)} .
$$

Consequently

$$
\begin{gathered}
\operatorname{res}_{\lambda=-\frac{n+|\gamma|}{2}-k}\left(P_{\gamma,+}^{\lambda}, \varphi\right)_{\gamma}=\operatorname{res}_{\lambda=-\frac{n+|\gamma|}{2}-k}\left(P_{\gamma,+}^{\lambda+k}, L_{B}^{k} \varphi\right)_{\gamma} \times \\
\times\left.\frac{1}{2^{2 k}(\lambda+1) \ldots(\lambda+k)\left(\lambda+\frac{n+|\gamma|}{2}\right) \ldots\left(\lambda+\frac{n+|\gamma|}{2}+k-1\right)}\right|_{\lambda=-\frac{n+|\gamma|}{2}-k},
\end{gathered}
$$

and

$$
\operatorname{res}_{\lambda=-\frac{n+|\gamma|}{2}-k}\left(P_{\gamma,+}^{\lambda+k}, L_{B}^{k} \varphi\right)_{\gamma}=\operatorname{res}_{\lambda=-\frac{n+|\gamma|}{2}}\left(P_{\gamma,+}^{\lambda}, L_{B}^{k} \varphi\right)_{\gamma}
$$


Therefore if $p+\left|\gamma^{\prime}\right|$ is even this residue vanishes. If $p+\left|\gamma^{\prime}\right|$ is not integer or $p+\left|\gamma^{\prime}\right| \in \mathbb{N}$ and $p+\left|\gamma^{\prime}\right|=2 k-1, k \in \mathbb{N}$ then (27) gives

$$
\operatorname{res}_{\lambda=-\frac{n+|\gamma|}{2}-k}\left(P_{\gamma,+}^{\lambda}, \varphi\right)_{\gamma}=\frac{(-1)^{\frac{q+\left|\gamma^{\prime \prime}\right|}{2}}}{2^{n+2 k} k !} \frac{\prod_{i=1}^{n} \Gamma\left(\frac{\gamma_{i}+1}{2}\right)}{\Gamma\left(\frac{n+|\gamma|}{2}+k\right)}\left(L_{B}^{k} \delta_{\gamma}(x), \varphi\right)_{\gamma} .
$$

This completes the proof of Theorem 2 .

Theorem 3. If $n+|\gamma|$ is even and $p+\left|\gamma^{\prime}\right|$ and $q+\left|\gamma^{\prime \prime}\right|$ are also even, $k \in \mathbb{N} \cup\{0\}$, then function $P_{\gamma,+}^{\lambda}$ has a simple pole in $\lambda=-\frac{n+|\gamma|}{2}-k$ with residue

$$
\begin{gathered}
\underset{\substack{\mathrm{res} \\
\lambda=-\frac{n+|\gamma|}{2}-k}}{P_{\gamma,+}^{\lambda}}=\frac{1}{\Gamma\left(\frac{n+|\gamma|}{2}+k\right)}\left[(-1)^{\frac{n+|\gamma|}{2}+k-1} \delta_{\gamma, 1}^{\left(\frac{n+|\gamma|}{2}+k-1\right)}(P)+\right. \\
\left.+\frac{(-1)^{\frac{q+\left|\gamma^{\prime \prime}\right|}{2}}}{2^{2 k} k !} \prod_{i=1}^{n} \Gamma\left(\frac{\gamma_{i}+1}{2}\right) L_{B}^{k} \delta_{\gamma}(x)\right] .
\end{gathered}
$$

If $p+\left|\gamma^{\prime}\right|$ and $q+\left|\gamma^{\prime \prime}\right|$ are not integer or $p+\left|\gamma^{\prime}\right|, q+\left|\gamma^{\prime \prime}\right| \in \mathbb{N}$ and $p+\left|\gamma^{\prime}\right|=$ $=2 m-1, q+\left|\gamma^{\prime \prime}\right|=2 k-1, m, k \in \mathbb{N}$ then function $P_{\gamma,+}^{\lambda}$ a pole of order two at $\lambda=-\frac{n+|\gamma|}{2}-k$. Coefficients $c_{-2}^{(k)}$ and $c_{-1}^{(k)}$ of expansion of function $P_{\gamma,+}^{\lambda}$ in Laurent series at $\lambda=-\frac{n+|\gamma|}{2}-k$ are expressed by formulas

$$
\begin{gathered}
c_{-1}^{(0)}=\frac{1}{\Gamma\left(\frac{n+|\gamma|}{2}+k\right)}\left[(-1)^{\frac{n+|\gamma|}{2}+k-1} \delta_{\gamma, 1}^{\left(\frac{n+|\gamma|}{2}+k-1\right)}(P)+\frac{(-1)^{\frac{n+|\gamma|}{2}}-1}{2^{2 k} k !} \times\right. \\
\left.\times \prod_{i=1}^{n} \Gamma\left(\frac{\gamma_{i}+1}{2}\right) \sin \left(\frac{p+\left|\gamma^{\prime}\right|}{2} \pi\right)\left(\psi\left(\frac{p+\left|\gamma^{\prime}\right|}{2}\right)-\psi\left(\frac{n+|\gamma|}{2}\right)\right) L_{B}^{k} \delta_{\gamma}(x)\right], \\
c_{-2}^{(k)}=(-1)^{\frac{n+|\gamma|}{2}+1} \frac{\sin \frac{\pi\left(p+\left|\gamma^{\prime}\right|\right)}{2} \prod_{i=1}^{n} \Gamma\left(\frac{\gamma_{i}+1}{2}\right)}{2^{n+2 k} k ! \pi \Gamma\left(\frac{n+|\gamma|+k}{2}\right)} L_{B}^{k} \delta_{\gamma}(x),
\end{gathered}
$$

where $\psi(x)=\frac{\Gamma^{\prime}(x)}{\Gamma(x)}$. 
Proof. Let $n+|\gamma|$ be even and $\lambda=-\frac{n+|\gamma|}{2}-k, k \in \mathbb{N} \cup\{0\}$. We express this $\left(P_{\gamma,+}^{\lambda}, \varphi\right)_{\gamma}$ in the form

$$
\left(P_{\gamma,+}^{\lambda}, \varphi\right)_{\gamma}=\frac{1}{\lambda+k} \int_{0}^{\infty} u^{\lambda+\frac{n+|\gamma|}{2}-1} \Phi_{0}(u) d u+\int_{0}^{\infty} u^{\lambda+\frac{n+|\gamma|}{2}-1} \Phi_{1}(\lambda, u) d u
$$

where $\Phi_{0}(u)=\operatorname{res}_{\lambda=-\frac{n+|\gamma|}{2}-k} \Phi(\lambda, u)$ and $\Phi_{1}(\lambda, u)$ is a regular at $\lambda=-\frac{n+|\gamma|}{2}-k$ function. By virtue of the proposal each integral in (30) may have at $\lambda=-\frac{n+|\gamma|}{2}-k$ a simple pole therefore function $\left(P_{\gamma,+}^{\lambda}, \varphi\right)_{\gamma}$ may have a pole of order two at $\lambda=-\frac{n+|\gamma|}{2}-k$. In the neighborhood of such a point we may expand $P_{\gamma,+}^{\lambda}$ in the Laurent series

$$
P_{\gamma,+}^{\lambda}=\frac{c_{-2}^{(k)}}{\left(\lambda+\frac{n+|\gamma|}{2}+k\right)^{2}}+\frac{c_{-1}^{(k)}}{\lambda+\frac{n+|\gamma|}{2}+k}+\ldots
$$

Let us find $c_{-1}^{(k)}, c_{-2}^{(k)}$. We have

$$
\left(c_{-2}^{(k)}, \varphi\right)_{\gamma}=\operatorname{res}_{\lambda=-\frac{n+|\gamma|}{2}-k} \int_{0}^{\infty} u^{\lambda+\frac{n+|\gamma|}{2}-1} \Phi_{0}(u) d u=\frac{1}{k !} \Phi_{0}^{(k)}(0) .
$$

If $k=0$ then $c_{-2}^{(0)}=\Phi_{0}(0)$. According to 13

$$
\begin{aligned}
\Phi_{0}(0) & =\frac{1}{4} \psi_{1}(0,0) \underset{\lambda=-\frac{n+|\gamma|}{2}}{\operatorname{res}} \int_{0}^{1}(1-t)^{\lambda} t^{\frac{q+\left|\gamma^{\prime \prime}\right|-2}{2}} d t= \\
& =\psi_{1}(0,0) \underset{\lambda=-\frac{n+|\gamma|}{2}}{\operatorname{res}} \frac{\Gamma\left(\frac{q+\left|\gamma^{\prime \prime}\right|}{2}\right) \Gamma(\lambda+1)}{4 \Gamma\left(\lambda+\frac{q+\left|\gamma^{\prime \prime}\right|}{2}+1\right)} .
\end{aligned}
$$

Considering that $\psi_{1}(0,0)=\varphi(0)\left|S_{1}^{+}(p)\right|_{\gamma^{\prime}}\left|S_{1}^{+}(q)\right|_{\gamma^{\prime \prime}}$ where $\left|S_{1}^{+}(p)\right|_{\gamma^{\prime}}$ and $\left|S_{1}^{+}(q)\right|_{\gamma^{\prime \prime}}$ were determined in 26 we obtain

$$
\begin{gathered}
\left(c_{-2}^{(0)}, \varphi\right)_{\gamma}= \\
=\frac{(-1)^{\frac{n+|\gamma|}{2}+1} B\left(\frac{p+\left|\gamma^{\prime}\right|}{2}, \frac{q+\left|\gamma^{\prime \prime}\right|}{2}\right)}{4 \pi} \sin \frac{\pi\left(p+\left|\gamma^{\prime}\right|\right)}{2}\left|S_{1}^{+}(p)\right|_{\gamma^{\prime}}\left|S_{1}^{+}(q)\right|_{\gamma^{\prime \prime}} \varphi(0) .
\end{gathered}
$$


When $p+\left|\gamma^{\prime}\right|$ is even (in this case $q+\left|\gamma^{\prime \prime}\right|$ is also even) we have $c_{-2}^{(k)}=0$ i.e. function $\left(P_{\gamma,+}^{\lambda}, \varphi\right)_{\gamma}$ has a simple pole at $\lambda=-\frac{n+|\gamma|}{2}$. If $p+\left|\gamma^{\prime}\right|$ is not integer or $p+\left|\gamma^{\prime}\right| \in \mathbb{N}$ and $p+\left|\gamma^{\prime}\right|=2 k-1, k \in \mathbb{N}$ then

$$
c_{-2}^{(0)}=(-1)^{\frac{n+|\gamma|}{2}+1} \frac{\sin \frac{\pi\left(p+\left|\gamma^{\prime}\right|\right)}{2} \prod_{i=1}^{n} \Gamma\left(\frac{\gamma_{i}+1}{2}\right)}{2^{n} \pi \Gamma\left(\frac{n+|\gamma|}{2}\right)} \delta_{\gamma}(x) .
$$

As well as in Theorem 2 we obtain that if $p+\left|\gamma^{\prime}\right|$ and $q+\left|\gamma^{\prime \prime}\right|$ are even then function $P_{\gamma,+}^{\lambda}$ has a simple pole at $\lambda=-\frac{n+|\gamma|}{2}-k$. If $p+\left|\gamma^{\prime}\right|$ and $q+\left|\gamma^{\prime \prime}\right|$ are not integer or $p+\left|\gamma^{\prime}\right|, q+\left|\gamma^{\prime \prime}\right| \in \mathbb{N}$ and $p+\left|\gamma^{\prime}\right|=2 m-1, q+\left|\gamma^{\prime \prime}\right|=2 k-1, m, k \in \mathbb{N}$ then

$$
c_{-2}^{(k)}=(-1)^{\frac{n+|\gamma|}{2}+1} \frac{\sin \frac{\pi\left(p+\left|\gamma^{\prime}\right|\right)}{2} \prod_{i=1}^{n} \Gamma\left(\frac{\gamma_{i}+1}{2}\right)}{2^{n+2 k} k ! \pi \Gamma\left(\frac{n+|\gamma|+k}{2}\right)} L_{B}^{k} \delta_{\gamma}(x) .
$$

Let's find $c_{-1}^{(k)}$. We have

$$
\begin{gathered}
\left(c_{-1}^{(k)}, \varphi\right)=\int_{0}^{\infty} u^{-k-1} \Phi_{0}(u) d u+ \\
+\underset{\lambda=-\frac{n+|\gamma|}{2}-k}{\operatorname{res}} \int_{0}^{\infty} u^{\lambda+\frac{n+|\gamma|}{2}-1} \Phi_{1}\left(-\frac{n+|\gamma|}{2}-k, u\right) d u .
\end{gathered}
$$

Since $\Phi_{0}(u)=\underset{\lambda=-k}{\operatorname{res}} \Phi(\lambda, u)$ then using the formulas 15 and 22 we obtain

$$
\int_{0}^{\infty} u^{-k-1} \Phi_{0}(u) d u=\frac{(-1)^{\frac{n+|\gamma|}{2}+k-1}}{\Gamma\left(\frac{n+|\gamma|}{2}+k-1\right)}\left(\delta_{\gamma, 1}^{\left(\frac{n+|\gamma|}{2}+k-1\right)}(P), \varphi\right)_{\gamma} .
$$

Thus

$$
\begin{gathered}
\operatorname{res}_{\lambda=-\frac{n+|\gamma|}{2}-k} \int_{0}^{\infty} u^{\lambda+\frac{n+|\gamma|}{2}-1} \Phi_{1}\left(-\frac{n+|\gamma|}{2}-k, u\right) d u= \\
=\left.\frac{1}{k !} \frac{\partial^{k} \Phi_{1}\left(-\frac{n+|\gamma|}{2}-k, u\right)}{\partial u^{k}}\right|_{u=0}=\left(\alpha_{\gamma}^{(k)}, \varphi\right)_{\gamma}
\end{gathered}
$$


and

$$
c_{-1}^{(k)}=\frac{(-1)^{\frac{n+|\gamma|}{2}+k-1}}{\Gamma\left(\frac{n+|\gamma|}{2}+k-1\right)} \delta_{\gamma, 1}^{\left(\frac{n+|\gamma|}{2}+k-1\right)}(P)+\alpha_{\gamma}^{(k)} .
$$

For $k=0$ we obtain

$$
\left(\alpha_{\gamma}^{(0)}, \varphi\right)_{\gamma}=\Phi_{1}\left(-\frac{n+|\gamma|}{2}, 0\right) .
$$

In order to find $\Phi_{1}\left(-\frac{n+|\gamma|}{2}, 0\right)$ we consider $\Phi(\lambda, 0)$. Using (24), 25) and 26) we obtain

$$
\Phi(\lambda, 0)=\varphi(0) \frac{\Gamma(\lambda+1) \prod_{i=1}^{n} \Gamma\left(\frac{\gamma_{i}+1}{2}\right)}{2^{n} \Gamma\left(\frac{p+\left|\gamma^{\prime}\right|}{2}\right) \Gamma\left(\lambda+\frac{q+\left|\gamma^{\prime \prime}\right|}{2}+1\right)} .
$$

Taking into account the formula $\Gamma(1-x) \Gamma(x)=\frac{\pi}{\sin \pi x}$ we can write

$$
\Phi(\lambda, 0)=\frac{\sin \pi\left(\lambda+\frac{q+\left|\gamma^{\prime \prime}\right|}{2}\right)}{\sin \pi \lambda} \frac{\Gamma\left(-\lambda-\frac{q+\left|\gamma^{\prime \prime}\right|}{2}\right) \prod_{i=1}^{n} \Gamma\left(\frac{\gamma_{i}+1}{2}\right)}{\Gamma\left(\frac{p+\left|\gamma^{\prime}\right|}{2}\right) \Gamma(-\lambda)} \varphi(0) .
$$

If $p+\left|\gamma^{\prime}\right|$ and $q+\left|\gamma^{\prime \prime}\right|$ are even then

$$
\lim _{\lambda \rightarrow-\frac{n+|\gamma|}{2}} \frac{\sin \pi\left(\lambda+\frac{q+\left|\gamma^{\prime \prime}\right|}{2}\right)}{\sin \pi \lambda}=(-1)^{\frac{q+\left|\gamma^{\prime \prime}\right|}{2}}
$$

hence function $\Phi(\lambda, 0)$ is regular at $\lambda=-\frac{n+|\gamma|}{2}$ and

$$
\Phi_{1}\left(-\frac{n+|\gamma|}{2}, 0\right)=\Phi\left(-\frac{n+|\gamma|}{2}\right)
$$

whence

$$
\left(\alpha_{\gamma}^{(0)}, \varphi\right)_{\gamma}=(-1)^{\frac{q+\left|\gamma^{\prime \prime}\right|}{2}} \frac{\prod_{i=1}^{n} \Gamma\left(\frac{\gamma_{i}+1}{2}\right)}{\Gamma\left(\frac{n+|\gamma|}{2}\right)} \varphi(0) .
$$

If $p+\left|\gamma^{\prime}\right|$ and $q+\left|\gamma^{\prime \prime}\right|$ are not integer or $p+\left|\gamma^{\prime}\right|, q+\left|\gamma^{\prime \prime}\right| \in \mathbb{N}$ and $p+\left|\gamma^{\prime}\right|=2 m-1$, $q+\left|\gamma^{\prime \prime}\right|=2 k-1, m, k \in \mathbb{N}$ then $\Phi(\lambda, 0)$ has a pole at $\lambda=-\frac{n+|\gamma|}{2}$. In this case

$$
\left(\alpha_{\gamma}^{(0)}, \varphi\right)_{\gamma}=\Phi_{1}\left(-\frac{n+|\gamma|}{2}, 0\right)=(-1)^{\frac{n+|\gamma|}{2}}-1 \prod_{i=1}^{n} \Gamma\left(\frac{\gamma_{i}+1}{2}\right) \times
$$




$$
\times \frac{\sin \left(\frac{p+\left|\gamma^{\prime}\right|}{2} \pi\right)\left(\psi\left(\frac{p+\left|\gamma^{\prime}\right|}{2}\right)-\psi\left(\frac{n+|\gamma|}{2}\right)\right)}{\Gamma\left(\frac{n+|\gamma|}{2}\right)} \varphi(0),
$$

where $\psi(x)=\frac{\Gamma^{\prime}(x)}{\Gamma(x)}$. We obtain

$$
c_{-1}^{(0)}=\frac{1}{\Gamma\left(\frac{n+|\gamma|}{2}\right)}\left[(-1)^{\frac{n+|\gamma|}{2}-1} \delta_{\gamma, 1}^{\left(\frac{n+|\gamma|}{2}-1\right)}(P)+\theta \delta_{\gamma}(x)\right],
$$

with a value

$$
\theta=(-1)^{\frac{q+\left|\gamma^{\prime \prime}\right|}{2}} \prod_{i=1}^{n} \Gamma\left(\frac{\gamma_{i}+1}{2}\right)
$$

if $p+\left|\gamma^{\prime}\right|$ and $q+\left|\gamma^{\prime \prime}\right|$ are even. If $p+\left|\gamma^{\prime}\right|$ and $q+\left|\gamma^{\prime \prime}\right|$ are not integer or $p+\left|\gamma^{\prime}\right|, q+\left|\gamma^{\prime \prime}\right| \in \mathbb{N}$ and $p+\left|\gamma^{\prime}\right|=2 m-1, q+\left|\gamma^{\prime \prime}\right|=2 k-1, m, k \in \mathbb{N}$ then

$$
\begin{gathered}
\theta=(-1)^{\frac{n+|\gamma|}{2}}-1 \prod_{i=1}^{n} \Gamma\left(\frac{\gamma_{i}+1}{2}\right) \sin \left(\frac{p+\left|\gamma^{\prime}\right|}{2} \pi\right) \times \\
\times\left(\psi\left(\frac{p+\left|\gamma^{\prime}\right|}{2}\right)-\psi\left(\frac{n+|\gamma|}{2}\right)\right) .
\end{gathered}
$$

Finally, in order to obtain $c_{-1}^{(k)}$ for arbitrary $k$, we again use the formula (29). This proves the desired result.

\section{References}

[1] Kipriyanov I. A., Ivanov L. A. Riezs potentials on the Lorentz spaces. Mat. sb., 1986, vol. 130(172), issue 4(8), pp. 465-474.

[2] Klyuchantsev M. I. Fractional order integrals and singular boundary problems. Differ. Uravn., 1976, vol. 12, issue 6, pp. 983-990.

[3] Guliyev V., Hasanov J. J. Necessary and sufficient conditions for the boundedness of B-Riesz potential in the B-Morrey spaces. Journal of Mathematical Analysis and Applications, 2012, vol. 347, issue 1, pp. 113-122. DOI: 10.1016/j.jmaa.2008.03.077.

[4] Aliev I. A., Gadzhiev A. D. Weighted estimates of multidimensional singular integrals generated by the generalized shift operator. Mat. Sb., 1992, vol. 183, issue 9, pp. 45-66. DOI: 10.1070/SM1994v077n01ABEH003428.

[5] Lyakhov L. N., Shishkina E. L. Inversion of general Riesz B-potentials with homogeneous characteristic in weight classes of functions. Doklady Mathematics, 2009, vol. 79, issue 3, pp. 377-381. 
[6] Shishkina E. L. Inversion of integral of B-potential type with density from $\Phi_{\gamma}$. Journal of Mathematical Sciences, 2009, vol. 160, issue 1, pp. 95-102.

[7] Kipriyanov I. A., Kononenko V. I. Fundamental solutions of some singular equations in partial derivatives. Differ. Uravn., 1969, vol. 5, issue 8, pp. 1470-1483.

[8] Stempak K., Ciaurri O. Transplantation and multiplier theorems for Fourier-Bessel expansions. Trans. Amer. Math. Soc., 2006, vol. 358, pp. 4441-4465.

[9] Yildiri H., Sarikaya M. Z. and Sermin O. The solutions of the n-dimensional Bessel diamond operator and the Fourier-Bessel transform of their convolution. Proc. Indian Acad. Sci. (Math. Sci.) Vol. 114, No. 4, November 2003, pp. 375-387.

[10] Kipriyanov I. A. Singular Elliptic Boundary Value Problems. Nauka, Moscow, Russia, 1997 (in Russian).

[11] Hörmander L. The Analysis of Linear Partial Differential Operators I. Springer-Verlag, 2003.

[12] Gelfand I. M., Shilov G. E. Generalised Functions. Academic Press, 1964.

[13] Lyakhov L. N., Shishkina E. L. Weighted mixed spherical means and singular ultrahyperbolic equation. Analysis (Germany), 2016, vol. 36, issue 2, pp. 65-70. DOI: 10.1515/anly-2015-5006.

[14] Lyakhov L. N., Polovinkin I. P. and Shishkina E. L. Formulas for the solution of the Cauchy problem for a singular wave equation with Bessel time operator. Doklady Mathematics, 2014, vol. 90, issue 3, pp. 737-742. DOI: $10.1134 / \mathrm{S} 106456241407028 \mathrm{X}$.

[15] Lyakhov L. N., Polovinkin I. P. and Shishkina E. L. On a Kipriyanov problem for a singular ultrahyperbolic equation. Differential Equations, 2014, vol. 50, issue 4, pp. 513-525. DOI: 10.1134/S0012266114040090.

[16] Shishkina E. L. Boundedness of potential operators with hyperbolic distance. Abstracts of the 8th International Workshop AMADE-2015, (Minsk, Belarus, September 14-19), 2015, Institute of Mathematics, National Academy of Sciences of Belarus, p. 90.

Received October 12, 2016.

In revised form, December 06, 2016.

Accepted December 06, 2016.

Voronezh State University

1, Universitetskaya pl., 394006 Voronezh, Russia

E-mail: ilina_dico@mail.ru 